\title{
Two New Heuristics for Two-Sided Bipartite Graph Drawing*
}

\author{
Matthew Newton ${ }^{1}$, Ondrej Sýkora ${ }^{1}$, and Imrich Vrto ${ }^{2}$ \\ 1 Department of Computer Science, Loughborough University \\ Loughborough, Leicestershire LE11 3TU, The United Kingdom \\ \{m.c.newton,o.sykora\}@lboro.ac.uk, Fax: +44 (0)1509 211586 \\ http://parc.lboro.ac.uk/ \\ 2 Institute of Mathematics, Slovak Academy of Sciences \\ Dúbravská 9, 84235 Bratislava, Slovak Republic \\ vrto@savba.sk, Fax: +421259306522
}

\begin{abstract}
Two new heuristic strategies are studied based on heuristics for the linear arrangement problem and a stochastic hill-climbing method for the two-sided bipartite crossing number problem. These are compared to the standard heuristic for two-sided bipartite drawing based on iteration of the barycentre method. Our experiments show that they can efficiently find good solutions.
\end{abstract}

\section{Introduction}

Graph drawing addresses the problem of finding a layout of a graph that satisfies given aesthetic and readability objectives. One standard problem with the drawing of bipartite graphs is that of two layer automatic drawing where the two vertex partitions are put in distinct points on two parallel lines and edges are drawn as straight line segments between the two lines. This type of drawing is the basic building block used for drawing hierarchical graphs [5/14]20] or producing row-based VLSI layouts [17. The most important objective is probably minimisation of the number of crossings in the drawing, as the aesthetics and readability of graph drawings depend on the number of edge crossings (see [16]). VLSI layouts containing less crossings are more easily realisable and consequently cheaper. There are two basic variants of the problem: one-sided and two-sided crossing minimisation. In the one-sided problem the vertices of one part of the bipartite graph are placed in fixed positions on one line. The vertices of the other part are placed on the other line and their positions are found so that the number of pairwise edge crossings is minimised. The two-sided bipartite crossing number problem (or just the bipartite crossing number problem) is if the vertices on both sides are not restricted. Unfortunately, both problems are NP-hard [7.8]. A lot of different methods have been designed to solve the one-sided problem - heuristics

\footnotetext{
* Research of all the authors was supported by the EPSRC grant GR/R37395/01. Research of the last two authors was supported by the Slovak Scientific Grant Agency grant No. 2/7007/20.
} 
and approximation algorithms (see e.g. 14]). For the two-sided bipartite problem a common technique is to apply a one-sided method iteratively. In 10 the one-sided methods, barycentre, LR-Opt (branch and cut method of [10]), split, median, stochastic, greedy-switch, assign and greedy-insert were applied iteratively until there was no change, i.e. until a local minimum had been achieved. The resulting ranking of the heuristics was as above. The iterated barycentre method was the best among the tested methods both in terms of quality and computation time. An interesting observation by the authors of [10, for graphs of up to about 15 vertices on both sides, was that for the graphs with a density of $10 \%$, the resulting number of crossings were 5 to 33 times larger than the optimal value.

In this paper we suggest two new heuristic strategies. The first is based on the result in 18. showing a strong relation between the linear arrangement problem of a bipartite graph and its bipartite crossing number. We used two different methods to find a solution to the linear arrangement problem which implies respective permutations of vertices on both sides (layers). One (linear arrangement based on Fiedler's vector: LAF-method) is based on computing the graph's second laplacian eigenvalue and its corresponding vector, usually called Fiedler's vector [9]. The other (linear arrangement based on Koren and Harel's method: LAKH-method) is based on the multiscale method of Koren and Harel [12]. The second strategy, stochastic hill-climbing ( $\mathrm{SH}$ )-method, is based on hill-climbing, a standard optimisation technique, and randomised swapping of vertices. In comparison with genetic algorithms or simulated annealing, stochastic hill-climbing is simpler and faster. Our method is different from the method of [3], which is also called 'stochastic' in [10. It also differs from the greedy-switch heuristic of [6] and from sifting [13] which are based on the swapping of neighbouring vertices.

In our experiments we compared the above three methods with the iterated barycentre (IB)-method. The results show that both LA*-methods usually outperform the IB-method, especially LAKH-method, which produces good drawings in an acceptable running time. The sH-method often produces the best results (see Table 1, shown instead of a graphical comparison due to lack of space).

\section{Notations}

Let $G=(V, E), V=V_{0} \cup V_{1}$ be a bipartite graph with vertex partitions $V_{0}$ and $V_{1}$. A bipartite drawing of $G$ is obtained by placing the vertices of $V_{0}$ and $V_{1}$ into distinct points on two horizontal lines $y_{0}$ and $y_{1}$, respectively, and drawing each edge with one straight line segment. Any bipartite drawing of $G$ is identified by two permutations $\pi_{0}$ and $\pi_{1}$ of the vertices on $y_{0}$ and $y_{1}$. The problem of the two-sided bipartite drawing of $G$ is to find permutations $\pi_{0}$ and $\pi_{1}$ that minimise the number of pairwise edge crossings in the corresponding bipartite drawing. The problem of the one-sided bipartite drawing of $G$ is the same, except the permutation $\pi_{1}$ is fixed. 
Let $\operatorname{bcr}\left(G, \pi_{0}, \pi_{1}\right)$ denote the total number of crossings in the bipartite drawing represented by the permutations $\pi_{0}$ and $\pi_{1}$. The bipartite crossing number of $G$, denoted by $\operatorname{bcr}(G)$, is the minimum number of crossings over all $\pi_{0}$ and $\pi_{1}$. Clearly, $\operatorname{bcr}(G)=\min _{\pi_{0}, \pi_{1}} \operatorname{bcr}\left(G, \pi_{0}, \pi_{1}\right)$.

Given an arbitrary graph $G=(V, E)$, the linear arrangement problem is to determine a bijection $f: V \rightarrow\{1,2,3, \ldots,|V|\}$ such that $\sum_{u v \in E}|f(u)-f(v)|$ is minimised. This minimum value, whose computation is NP-hard [8], is denoted by $\mathrm{L}(G)$.

Furthermore, $d_{v}$ denotes the degree of $v, \delta_{G}$ the minimum degree of $G$ and $\Delta_{G}$, the maximum degree of $G$.

\section{Linear Arrangement Problem Based Methods}

The bipartite crossing number problem was studied in [18] and a connection between this problem and the linear arrangement problem was established. Lower and upper bounds for bipartite crossing number $\operatorname{bcr}(G)$ were derived, where the main term is the optimal arrangement value:

Theorem 1 Let $G=\left(V_{0}, V_{1}, E\right)$, then

$$
\frac{1}{36} \delta_{G} \mathrm{~L}(G)-\frac{1}{12} \sum_{v \in V} d_{v}^{2} \leq \operatorname{bcr}(G) \leq 5 \Delta_{G} \mathrm{~L}(G) .
$$

By using this result, a polynomial time approximation algorithm [18] with performance guarantee $\mathrm{O}(\log n)$ from the optimal was obtained for the bipartite crossing number problem if $\Delta_{G}=O\left(\delta_{G}\right)$. The algorithm is based on an approximation algorithm for the linear arrangement problem, where an approximated solution of the linear arrangement problem is found and then the vertices of the partitions of $V_{0}$ and $V_{1}$ are put on the lines $y_{0}$ and $y_{1}$ in the same order as produced for the linear arrangement problem. Using this idea, we can use any heuristic that solves the linear arrangement problem to produce the pair of permutations $\pi_{0}$ and $\pi_{1}$.

In [9] a heuristic is suggested that solves the linear arrangement problem by computing the corresponding (so called Fiedler's) eigenvector $f$ to the smallest positive Laplacian eigenvalue $\lambda_{G}$ of the graph $G$. Recall that the Laplacian of a graph $G$ is the matrix $I(G)-A(G)$, where $A(G)$ is the adjacency matrix of the graph $G$ and $I(G)$ is the diagonal matrix with vertex degrees on the diagonal, i.e. $i_{v v}=d_{v}$, and $i_{u v}=0$ if $u \neq v$ and the Fiedler's eigenvector $f$ fulfills: $(I(G)-A(G)) f=\lambda_{G} f$. Using this approximation of linear arrangement we get the LAF-method shown in Algorithm 1.

The other method (LAKH-method) is based on the multiscale method of Koren and Harel 12 that computes an approximation of the linear arrangement.

\section{Stochastic Hill-Climbing Method}

The space of orderings of vertices of both sides for two-sided bipartite drawing problem can be represented by all pairs of permutations of $\left\{0,1,2, \ldots, n_{0}-1\right\}$, 
Algorithm 1. "Linear Arrangement based on Fiedler's vector" (LAF-method) find smallest positive Laplacian eigenvalue $\lambda_{G}$ or a good approximation find corresponding eigenvector (Fiedler vector) construct approximation to the solution of the Linear Arrangement Problem construct permutations $\pi_{0}$ and $\pi_{1}$

evaluate the number of crossings for the pair of permutations

and of $\left\{0,1,2, \ldots, n_{1}-1\right\}$, where $n_{0}$ and $n_{1}$ are the number of vertices in $V_{0}$ and $V_{1}$, respectively. To find a solution of the two-sided bipartite drawing problem means searching this space for a pair of permutations providing the minimum number of crossings. To move across the space we might use some type of steps, evaluate a new visited pair of permutations, accept it if it fulfills a condition (e.g. it has lower or equal number of crossings) and continue to search from the new accepted permutation. If the new permutation was not accepted we continue from the old one. The starting permutations are given by the input. Algorithm 2 shows the specific method we used.

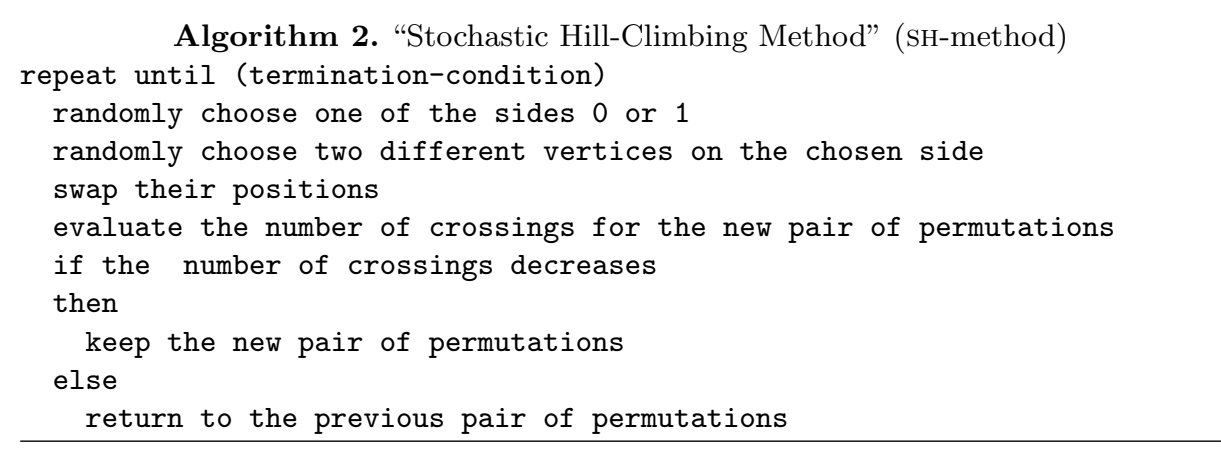

The termination condition can be defined by the number of iterations, or the number of iterations that have passed since the last swap that gave an improvement to the number of crossings (the number of stagnations). According to our experience, the termination should happen if the number of stagnations exceeds a value $\alpha *$ density $/($ density $+\beta)$ where density $=|E| /|V|$, and $\alpha, \beta$ are proper constants. For the sizes of our graphs these constants could be: $\alpha=$ $1350, \beta=65$.

In our experiments we ran the sH-method while the number of crossings was higher than that achieved by the IB-method, the LAF-method and LAHK-method. The sH-method program also had a maximum time limit of five minutes and a few graphs did not complete within this limit. For some graphs SH-method stagnated and did not reach the number of crossings of the IB (this includes e.g. leonardo-90x64 and hyper-9) or LA*-methods (this includes e.g. bcspwr01 and 
cycle-100). An asterisk in Table 1 indicates one of the previous two conditions. Some graphs completed in less than 0.00005 second, so due to rounding it looks

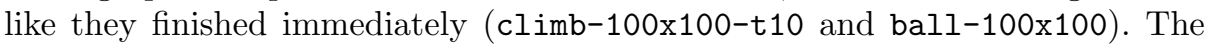
number of crossings that each algorithm reached, together with the time taken, is shown in Table 1; IB-method in columns two and three, LAF-method in columns five and six, LAKH-method in columns eight and nine. The running times of SHmethod necessary to achieve the number of crossings equal to or below that of the IB-, LAF- and LAKH-methods are shown in columns four, seven and ten, respectively. The best number of crossings found for each graph is shown in boldface.

\section{Experiments}

Our algorithms were implemented in C, running on 50 Sun ULTRAsparc 5 workstations running Solaris 7 . The algorithms were compiled with the GNU C compiler version 2.95.2. One of the computers ran a job control system, written in Perl, to run experiments continuously on the other machines.

The experiments for the IB-, LAF- and LAKH-methods were run first. These programs output the pair of permutations of the nodes in each graph, $\pi_{0}$ followed by $\pi_{1}$, from which the number of crossings was counted. Before each experiment, $\pi_{0}$ and $\pi_{1}$ were randomised (this did not affect the LA*-methods, which do not need an initial permutation).

The quality of the results of the LA*-methods depends on the quality of the approximation of $\mathrm{L}(G)$ for a graph $G$. To compute the Fiedler's eigenvector in the LAF-method we used the procedure jacobi from Numerical Recipes [15]. Although the jacobi procedure is not too efficient, it gives trustworthy results and is recommended for problems of small-to-moderate order. Typical $n \times n$ matrices require $12 n^{3}$ to $20 n^{3}$ operations. In the LAKH-method we used the multiscale algorithm of Koren and Harel [12] for solving the linear arrangement problem running in linear time in the graph size $(|V|+|E|)$. Apart from meshes, the LAKH-method gave better results than the LAF-method. It is also much faster, especially for larger graphs. Thanks are due to Yehuda Koren who kindly provided us their procedure so we were able to test this method.

All IB-, LAF- and LAKH-methods were run ten times on each graph and the average number of crossings and running time were recorded.

A new set of experiments were then run for the SH-method. The algorithm was run thirty times for each graph. For the first, second and third ten runs the program halted when the number of crossings was equal to or below that of the IB-, LAF-, LAKH-methods, respectively. As in the first set of experiments, $\pi_{0}$ and $\pi_{1}$ of each graph were randomised before each test run.

Graph Test Sets. For our experiments we used the classes of graphs from [4] that contain 'synthetic' bipartite graphs with a known bipartite crossing number, bipartite graphs extracted from digitised images (originally suggested by Knuth in the Stanford Graphbase [11]) and from test data retrieved from US National Institute of Standards and Technology [2]. For our tests we also added 
the following graphs for which we either know, or believe we know, the optimum drawings. Among these are cycles (with bipartite crossing number $\frac{n}{2}-1$, if there are $n$ vertices in the cycle, $n$ is even), rectangular meshes, square $n \times n$ meshes (with supposed bipartite crossing number $\frac{4}{3} n(n-1)(n-2)+1$ ) and hypercubes (see [19]). Finally, we also used randomly generated graphs.

Random Graphs. The random graphs we used were generated based on edge density. The generator takes two arguments: $n$, the number of nodes the graph should have, and $p \in[0 . .100]$ that determines the percentage chance that an edge will exist between two nodes. $n$ must be an even number, and the graph is generated with $\frac{n}{2}$ nodes in each part. The general graphs were generated having sizes $n \in[20,40,60,80,100,200,400,600,800,1000]$ and densities of $10 \%$, $1 \%$ and $0.1 \%$.

\section{Acknowledgement}

The authors wish to thank Yehuda Koren and Irene Finocchi, for making available their software, and Mark Withall for useful discussions during the preparation of this paper.

Table 1. Comparisons between IB-method (columns two and three), LAF-method (columns five and six), LAKH-method (columns eight and nine) and sH-method. In columns four, seven and ten are the running times necessary for SH-method to achieve the number of crossings equal to or below that of the IB-, LAF- and LAKH-methods, respectively. Time is in seconds.

\begin{tabular}{|c|c|c|c|c|c|c|c|c|c|}
\hline \multirow[t]{2}{*}{ Graph Name } & \multicolumn{2}{|c|}{ IB-method } & \multirow{2}{*}{\begin{tabular}{|c} 
SH to \\
IB \\
Time
\end{tabular}} & \multicolumn{2}{|c|}{ LAF-method } & \multirow{2}{*}{$\begin{array}{l}\text { SH to } \\
\text { LAF } \\
\text { Time }\end{array}$} & \multicolumn{2}{|c|}{ LAKH-method } & \multirow{2}{*}{$\begin{array}{l}\text { SH to } \\
\text { LAKH } \\
\text { Time }\end{array}$} \\
\hline & Crossings & Time & & Crossings & Time & & Crossings & Time & \\
\hline$a-100 \times 100$ & 2550802 & 0.0031 & \begin{tabular}{|l|}
0.3989 \\
\end{tabular} & 2716464 & 19.3547 & 0.0111 & 1301275 & 15.888 & \\
\hline & 2414484 & 0.0025 & 0.8535 & 1327488 & 2.5816 & 25.6836 & 1301275 & 15.034 & \\
\hline $\mathrm{d} 20$ & 3621791 & 0.4223 & 0.0088 & 1177229 & 1296.39 & 51.7084 & 1021504 & 37.708 & \\
\hline andywarhol-100x65 & 1893498 & 0.0074 & & 2856377 & 2.248 & 11.4422 & 2784605 & 17.056 & 35.1931 \\
\hline ball-100×100 & 947575 & 0.004 & & 934566 & 9.413 & 0.0068 & 749982 & 9.038 & 22.3192 \\
\hline barpermute- $100 \times 100$ & 3175608 & 0.004 & 2.6106 & 4161703 & 5.4557 & 0.0162 & 2040676 & 18.829 & 61.6639 \\
\hline bars-100x100 & 3517232 & 0.0325 & 0.0324 & 2075771 & 6.7551 & & 2153777 & 21.607 & 45.9696 \\
\hline bcspwr01 & 2954 & 0.0019 & 0.0029 & & 0.2698 & * & 422 & 1.263 & \\
\hline tk 15 & 889155 & 0.1933 & & 727592 & 923.3729 & & 741208 & 36.756 & \\
\hline ick-100×100 & 501133 & 0.0171 & 0 & 473653 & 24.6587 & 0.0011 & 248575 & 10.123 & 3.3267 \\
\hline & 1559231 & 0.0078 & & 2076490 & & 1.2589 & 170 & 15.594 & 12.8803 \\
\hline & & 0.0026 & 0.0075 & 12788 & 1.1197 & & 12847 & 3.835 & \\
\hline ar-100x100 & 88815 & 0.0027 & & 88815 & & * & 88815 & 8.597 & 91.8295 \\
\hline cbar- $20 \times 20$ & 465 & 0.0004 & \begin{tabular}{|l|} 
\\
0.0018 \\
\end{tabular} & 19 & 0.0314 & & 19 & 0.515 & \\
\hline $50 \times 50$ & 3870 & 0.0015 & 19.2498 & 3870 & 0.6231 & 21.2854 & 3870 & 2.64 & 26.1418 \\
\hline $100 \times 100-\mathrm{t} 10$ & 19276 & 0.0077 & & 10824 & 10.7103 & 0.0212 & 4764 & 3.768 & \\
\hline $\mathrm{x} 100-\mathrm{t} 100$ & 544406 & 0.0066 & 0.0182 & 536316 & 29.5412 & 0.0359 & 252671 & 9.847 & \\
\hline & & 0.0071 & & & & & & 10.205 & 10.0924 \\
\hline & & & 0.0116 & & & & & & $\begin{array}{r}7.8663 \\
\end{array}$ \\
\hline & & 0.0093 & 0 & & & 0.0194 & & 12.783 & 6.3544 \\
\hline $100 \times 100-1140$ & 1355640 & 0.0138 & 0 & 1081502 & 5.6 & 0.4582 & 349 & 14.181 & 11.065 \\
\hline $100 \times 100-\mathrm{t} 150$ & 2627049 & 0.0155 & 0 & 1690572 & 5.7368 & 11.4668 & 1569210 & 17.33 & 20.2285 \\
\hline $100 \times 100-\mathrm{t} 160$ & 3796488 & 0.0297 & 0 & 2697887 & 5.6074 & 8.7222 & 2597067 & 19.739 & 20.7422 \\
\hline 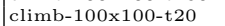 & 83505 & 0.0087 & & 39548 & 11.8 & 1.0374 & 35877 & 5.512 & 0.4004 \\
\hline & & & 0.0022 & & 14.7 & & & 6.53 & 0.8385 \\
\hline & 1698 & 0.0082 & 0.0087 & 100 & 15. & 0.7436 & & 6.581 & 0.5109 \\
\hline & & 0.0078 & & & & 0.0 & & 7.506 & 0.9283 \\
\hline & 23988 & 0.0067 & 0.0406 & 151225 & & 782 & & 7.294 & 2.0096 \\
\hline & & 0.0066 & & & & & & 7.927 & 1.025 \\
\hline $\mathrm{cl}$ & & 0.0076 & 0.16 & & & & & 199 & 1.1534 \\
\hline $100 \times 100-\mathrm{t} 90$ & 341906 & 0.0084 & 0.0457 & 224044 & 24.6957 & 0.7863 & 184132 & 8.31 & 2.4242 \\
\hline$-100 \times 100$ & 997171 & 0.0084 & & 914106 & 5.539 & 0.0136 & 471565 & 12.415 & 13.5789 \\
\hline & 1316 & 0.0004 & & 1084 & 0.0322 & 0.0004 & 536 & 0.659 & 0.0221 \\
\hline & 24032 & 0.0016 & 0.0001 & 19797 & & & 11464 & 2.31 & 0.1219 \\
\hline & 1319 & 0.004 & 0 & & & & & 6.069 & 1.0191 \\
\hline & 4157 & & 0 & & & & & 8.251 & \\
\hline & & 0.00 & & 49 & & & 83 & 1.53 & \\
\hline 1000 & 6639 & 1.13 & & 499 & 4875.714 & * & 778 & 17.825 & * \\
\hline-20 & 48 & 0.0001 & 0.0004 & 9 & 0.0 & & 13 & 0.168 & * \\
\hline & 1057 & 0.0198 & & 99 & 10.3766 & & 171 & 3.355 & * \\
\hline & 1813 & 0.054 & & 149 & 41.6743 & & 250 & 5.077 & \\
\hline
\end{tabular}




\begin{tabular}{|c|c|c|c|c|c|c|c|c|c|}
\hline \multirow[t]{2}{*}{ Graph Name } & \multicolumn{2}{|c|}{ IB-method } & SH to & LAF-m & ethod & SH to & LAKH-m & nethod & SH to \\
\hline & Crossings & Time & Time & Crossings & Time & Time & Grossings & Time & $\begin{array}{l}\text { LAKHe } \\
\text { Time }\end{array}$ \\
\hline cyclo -40 & 122 & 0.0005 & 0.0053 & 19 & 0.0457 & & 29 & 0.474 & \\
\hline cycle -400 & 2228 & 0.0981 & & 199 & 152.9575 & * & 328 & 7.116 & $*$ \\
\hline cycle -500 & 2987 & 0.2015 & & 249 & 282.9466 & * & 404 & 8.998 & $*$ \\
\hline cycle -60 & 274 & 0.0014 & 0.0104 & 29 & 0.1782 & * & 45 & 0.8 & $*$ \\
\hline cycle-80 & 253 & 0.0024 & 0.0684 & 39 & 0.4786 & * & 63 & 1.185 & * \\
\hline geom $2-100 \times 100$ & 179880 & 0.0037 & 0.1256 & 391832 & 2.4829 & 0 & 43073 & 6.538 & $*$ \\
\hline hyper-10 & 2221867 & 0.2571 & & 2548526 & 7116.157 & 144.4579 & & & \\
\hline hyper-4 & 131 & 0.0001 & 0.0011 & 118 & 0.0049 & 0.0022 & 124 & 0.154 & 0.0011 \\
\hline hyper -5 & 999 & 0.0002 & 0.0018 & 776 & 0.0346 & 0.0143 & 786 & 0.545 & 0.0093 \\
\hline hyper-6 & 4710 & 0.0006 & 0.0395 & 4200 & 0.1669 & & 4359 & 1.494 & 0.0845 \\
\hline hyper-7 & 22714 & 0.0026 & & 23464 & 16.9141 & 0.526 & 22134 & 3.957 & 1.2749 \\
\hline hyper-8 & 108038 & 0.013 & * & 106400 & 38.4887 & & 106922 & 9.756 & 9.6853 \\
\hline hyper-9 & 494357 & 0.0541 & & 535292 & 1247.529 & 24.5621 & 498715 & 21.38 & 70.8947 \\
\hline irregular-100×100 & 886963 & 0.0027 & 6.6072 & 3690604 & 5.2365 & 0 & 492835 & 12.699 & \\
\hline ladyshoe- $67 \times 100$ & 883543 & 0.0054 & 0.9114 & 727351 & 2.0314 & 2.7296 & 669064 & 11.151 & 13.8607 \\
\hline & 455396 & 0.0077 & & 1341314 & 1.8802 & 0.3251 & 1073758 & 11.988 & 4.9457 \\
\hline ndas & 1318184 & 0.8055 & 0.0013 & 1266715 & 836.6369 & $299.3661 \mid$ & 736 & 25.755 & $*$ \\
\hline mesh-10-10 & 1212 & 0.0028 & & 997 & 0.7666 & & 1009 & 1.978 & \\
\hline esh-15-15 & 6286 & 0.0175 & & 3897 & 23.6179 & & 3858 & 5.614 & \\
\hline $\operatorname{esh}-20-20$ & 12570 & 0.0701 & * & 9519 & 118.2442 & * & 9993 & 8.964 & \\
\hline esh- $25-25$ & 30245 & 0.2926 & * & 19340 & 551.8628 & * & 19708 & 14.722 & * \\
\hline$e s \mathrm{~h}-30-10$ & 6246 & 0.0445 & * & 3729 & 31.9283 & * & 3367 & 6.287 & $*$ \\
\hline & 16014 & 0.1238 & * & 8866 & 182.8301 & * & 8119 & 10.96 & * \\
\hline & 27610 & 0.2655 & * & 16465 & 650.1603 & * & 14920 & 13.7 & * \\
\hline esh-30-25 & 39139 & 0.5404 & * & 25258 & 1056.526 & * & 23524 & 18.843 & \\
\hline esh-30-30 & 45950 & 0.654 & & 34200 & 2470.742 & $*$ & 34836 & 23.848 & \\
\hline hh $-30-5$ & 1225 & 0.0066 & * & 706 & 2.3688 & * & 679 & 2.946 & \\
\hline $35-35$ & 85203 & 1.5665 & & 55098 & 6805.752 & * & & & $*$ \\
\hline & 120812 & 3.2542 & & 84679 & 21971.12 & * & * & & $*$ \\
\hline & & 0.0002 & 0.0007 & & 0.0088 & * & 90 & 0.302 & 0.0124 \\
\hline$a-100 \times 65$ & 1945030 & 0.0101 & $0.000 *$ & $\begin{array}{r}5179482 \\
512\end{array}$ & $\begin{array}{l}2.7837 \\
\end{array}$ & 0.4914 & 4314354 & $\begin{array}{r}19.7766 \\
\end{array}$ & 93.816 \\
\hline & & 0.0003 & & 6332 & 0.021 & & 6156 & 0.919 & $*$ \\
\hline & 50578 & 0.0023 & & 128954 & 0.153 & 0.0044 & 106336 & 3.903 & \\
\hline onnalisa-60×39 & 257956 & 0.0035 & & 656433 & 0.5054 & 0.0472 & 540595 & 7.249 & 11.2691 \\
\hline talisa-80x52 & 770234 & 0.0077 & & 2072231 & 1.3043 & 0.2279 & 1731778 & 12.19 & 56.0786 \\
\hline rface $-100 \times 100$ & 2146803 & 0.0175 & 0.7537 & 1807232 & 5.771 & 3.1508 & 1599527 & 16.847 & 36.2476 \\
\hline oran & 4941969 & 0.9359 & 0.0601 & 2202672 & 710.6326 & 26.2889 & 1955821 & 36.533 & 88.4403 \\
\hline & $2.05 e+09$ & 0.265 & 73.6012 & $1.91 \mathrm{e}+09$ & 608.8471 & & $1.85 e+09$ & 423.455 & \\
\hline $\begin{array}{r}r \\
z\end{array}$ & & 0.0005 & & & 0.1306 & & 0 & & 0 \\
\hline & 102 & & 0.0001 & 102 & & & 0 & 1.201 & $*$ \\
\hline & 16707 & 0.0074 & 0.0003 & 9575 & 0.7139 & 0.0667 & 8304 & 2.706 & 1.2585 \\
\hline lom-100 & 19093 & 0.3769 & 0.0049 & 18980 & 3.6272 & 0.0039 & 0 & & \\
\hline$m-100$ & 1683123 & 9.4952 & 0.0113 & 1672515 & 3937.91 & 0.009 & 772973 & 41.513 & * \\
\hline & $1.58 \mathrm{e}+08$ & 6.0268 & 1.243 & $1.4 \mathrm{e}+08$ & 3973.301 & 197.7546 & $1.36 e+08$ & 226.149 & \\
\hline & 0 & 0.0001 & 0 & 0 & 0.0001 & $\begin{array}{ll}1 & 0\end{array} \mid$ & 0 & 0 & \\
\hline & 0 & 0.00 & & 0 & 0.0001 & & 0 & 0.075 & 0 \\
\hline & 25 & & & 12 & & & 0 & 0.126 & \\
\hline & 5 & 0.0016 & 0.0006 & 5 & 0.4618 & 0.0011 & 0 & 0 & 0.0092 \\
\hline$n-20$ & 2257 & 0.0119 & & 1748 & 0.1166 & 0.0017 & 0 & & \\
\hline$n-20$ & 275147 & 0.0446 & 0.0011 & 201022 & 6.843 & 0.4308 & 182662 & 9.832 & 3.8573 \\
\hline & & 0.0001 & & 1 & 0.0003 & & & 0.286 & 0.0001 \\
\hline & & 0.0001 & & 1 & 0.0106 & 0.0003 & 0 & 0.375 & 0.0004 \\
\hline & 370 & 0.0004 & 0.0002 & 268 & 0.0217 & 0.0006 & 87 & 0.498 & $*$ \\
\hline & 400 & 0.0154 & 0.0006 & 288 & 0.6268 & & 0 & 0 & * \\
\hline & 42140 & & & 21995 & 234.6 & & 5032 & 7.745 & $*$ \\
\hline & 4210581 & 0.6203 & 0.0424 & 3435193 & 118.4158 & 5.4909 & 3215659 & 35.652 & 41.3217 \\
\hline & & 0.0002 & & 0 & 0.0261 & & & & \\
\hline & 13 & 0.00 & 0.0001 & 8 & 0.0263 & 0.0004 & & 0.594 & 0.0021 \\
\hline & 1798 & 0.0015 & & 2018 & 0.164 & & 376 & 1.011 & \\
\hline & 2806 & 0.1033 & & 2497 & 0.92 & 0.0035 & 0 & & \\
\hline & 204855 & 0.8702 & 0.0043 & 206670 & 611.0068 & 0.001 & 58159 & 15.037 & * \\
\hline & 20385097 & 2.5 & 0.0854 & 17455934 & 715.8014 & 299.6423 & 16400000 & 1.408 & * \\
\hline & & & & & & & & & \\
\hline & 31 & 0.0005 & 0.0002 & 15 & 0.0151 & 0.0011 & 0 & 0.9 & * \\
\hline & 5416 & 0.00 & & 2143 & & & 2011 & 1.673 & * \\
\hline & & & & & & 0.0036 & & & * \\
\hline & 620494 & 6.41 & & 555193 & 2080.685 & 0.0794 & 233566 & 25.781 & * \\
\hline & 64476577 & 3.8251 & 0.1718 & 56162991 & 2123.121 & 299.6723 & 53900000 & 140.667 & $*$ \\
\hline & 1376092 & 0.0053 & 0.0032 & 1389508 & 4.7603 & & 344728 & 11.287 & * \\
\hline & & & 0.0004 & & & & 338 & & * \\
\hline & 2754 & & & & & & & & 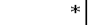 \\
\hline & & 0.00 & 1.0449 & & & & 275 & 15.098 & \\
\hline & & & & & & & & 9.185 & 414 \\
\hline & & & & & & & 303 & 21.7 & \\
\hline $2-100 x$ & 814985 & 0.0215 & 0.0005 & 432977 & 5.72 & 2.24 & 390889 & 11.83 & 13.0504 \\
\hline are3-100x 100 & 5035435 & 0.0327 & 0.0182 & 4238284 & 6.0099 & 7.0197 & 4024066 & 23.237 & 32.7983 \\
\hline & 2226143 & 0.0112 & & 1073482 & 5.5928 & 28.7785 & 1080003 & 15.952 & 40.5893 \\
\hline & & 0.0284 & 0.0003 & & 6.73 & & & & \\
\hline & & & 0.088 & & & & & & 50.4353 \\
\hline $0 \times 100$ & 2900151 & 0.0 & & 081 & & 3.2826 & & & 18.7751 \\
\hline$x 100$ & 3190315 & 0.00 & 0.2814 & 3184326 & 5.08 & 0.3251 & 2033573 & 16.586 & \\
\hline 00 & 6123591 & 0.0029 & 0.008 & 4003275 & 6.5481 & * & 4003275 & 20.285 & * \\
\hline zenios & 6845283 & 0.6242 & 1.0495 & 1113354 & 1220.934 & * & 987540 & 33.587 & \\
\hline
\end{tabular}




\section{References}

1. Bastert, O., Matuszewski, C.: Layered drawings of digraphs. In: Drawing Graphs, Methods and Models (eds. Kaufmann, M., Wagner D.), LNCS 2025, Springer (2001) 87-118

2. Boisvert, R., Pozo, R., Remington, K., Barrett, R., Dongarra, J.: Matrix Market: a web resource for test matrix collections. In: The Quality of $\mathrm{Nu}^{-}$ merical Software: Assessment and Enhancement (ed. Boisvert, R.), Chapman and Hall, London, (1997) 125-137, Matrix Market is available at the URL: http://math.nist.gov/MatrixMarket/

3. Dresbach, S.: A new heuristic layout algorithm for DAGs. In: Operations Research Proceedings 1994 (eds. Derigs, U., Drexl, A.B.A.), Springer (1994) 121-126

4. Demetrescu, C., Finocchi, I.: Removing cycles for minimizing crossings. J. Experimental Algorithmics, to appear

5. Di Battista, G., Eades, P., Tamassia, R., Tollis, I.G.: Graph Drawing: Algorithms for Visualization of Graphs. Prentice Hall (1999)

6. Eades, P., Kelly, D.: Heuristics for reducing crossings in 2-layered networks. Ars Combin. 21 (1986) 89-98

7. Eades, P., Wormald, N.: Edge crossings in drawings of bipartite graphs. Algorithmica 11 (1994) 379-403

8. Garey, M.R., Johnson, D.S.: Crossing number is NP-complete. SIAM J. Algebraic Disc. Meth. 4 (1983) 312-316

9. Juvan, M., Mohar, B.: Optimal linear labellings and eigenvalues of graphs. Discrete Appl. Math. 36 (1992) 153-168

10. Jünger, M., Mutzel, P.: 2-Layer straight line crossing minimization: Performance of exact and heuristic algorithms. J. Graph Algorithms Appl., 1 (1997) 1-25

11. Knuth, D.E.: The Stanford GraphBase: A platform for combinatorial computing. Addision-Wesley, (1993)

12. Koren, Y., Harel, D.: A multi-scale algorithm for the linear arrangement problem. In: 28th Intl. Workshop on Graph-Theoretic Concepts in Computer Science (WG'2002), LNCS, Springer (2002), to appear

13. Matuszewski, C., Schönfeld, R., Molitor, P.: Using sifting for $k$-layer straightline crossing minimization. In: 7th Intl. Symp. on Graph Drawing (GD'99), LNCS 1731, Springer (1999) 217-224

14. Mutzel, P.: Optimization in leveled graphs. In: Pardalos, M., Floudas C.A. (eds.): Encyclopedia of Optimization. Kluwer, Dordrecht (2001)

15. Press, W.H., Teukolsky, S.A., Vetterling, W.T., Flannery, B.P.: Numerical Recipes in C. The Art of Scientific Computing. Second edition, Cambridge University Press (1992) http://www.nr.com/

16. Purchase, H.: Which aesthetic has the greatest effect on human understanding? In: 5th Intl. Symp. on Graph Drawing (GD'97), LNCS 1353, Springer (1998) 248-261

17. Sarrafzadeh, M., Wong, C.K.: An Introduction to VLSI Physical Design. McGraw Hill, (1996)

18. Shahrokhi, F., Sýkora, O., Székely, L.A., Vrťo, I.: On bipartite drawings and the linear arrangement problem. SIAM J. Computing 30 (2001), 1773-1789

19. Shahrokhi, F., Sýkora, O., Székely, L.A., Vrťo, I.: A new lower bound for the bipartite crossing number with algorithmic applications. Theoretical Computer Science 245 (2000) 281-294

20. Sugiyama, K., Tagawa, S., Toda, M.: Methods for visual understanding of hierarchical system structures. IEEE Transactions on Systems, Man, and Cybernetics SMC-11 (1981) 109-125 